\section{Cytotoxicity of silicone oil on cultivated human corneal endothelium}

\section{Introduction}

Extended tamponade with silicone oil is useful in the treatment of complicated retinal detachment, such as proliferative vitreoretinopathy, giant retinal tear, and trauma. ${ }^{1}$ Although considered nontoxic, unfortunately, intraocular silicone oil causes late complications including cataract formation, glaucoma, subretinal infiltration, ${ }^{2,3}$ optic nerve damage, ${ }^{4-7}$ and silicone oil-associated keratopathy. The changes that derive from silicone oil-associated keratopathy include band keratopathy, corneal thinning, retro-corneal membrane formation, and irreversible corneal endothelium cell loss. ${ }^{8,9}$ It was hypothesized that the adverse effects of silicone oil on corneas are possibly related to forward migration of silicone oil to the anterior chamber and corneal touch. ${ }^{10,11}$ The silicone oil might act as a barrier separating human corneal endothelial cells (CEs) from the nutrition normally supplied by the aqueous humour. ${ }^{12}$ Nevertheless, the possible direct cytotoxic effect of silicone oil on CEs has not been fully evaluated because silicone oil has been assumed inert in ocular tissues. ${ }^{13}$

Animal studies in which silicone oil was injected into eyes have been performed to investigate the possible toxicity of silicone oil on ocular tissues, and histopathologic studies of silicone oil-filled human eyes have been reported. ${ }^{8,12}$ Evidence showed the silicone oil penetration into ocular tissues including the anterior segments, and although no massive inflammation has been detected in the zones in contact with silicone oil, immunoglobulins and complement fractions are present in the stroma and around the droplets of emulsified silicone oil, suggesting a local immune reaction. ${ }^{14}$ Furthermore, injection of silicone oil into rabbit's anterior chamber causes the changes in CE morphology and permeability; ${ }^{15,16}$ these evidences indicated the possible cytotoxic
Keywords: silicone oil; cornea endothelium; biocompatibility
Eye (2008) 22, 282-288; doi:10.1038/sj.eye.6702962; published online 24 August 2007 \\ Published online: 24 August \\ 2007 \\ Financial interest: None \\ Received: 11 December \\ 2006 \\ Accepted in revised form: \\ 22 July 2007 \\ ${ }^{1}$ Department of
Ophthalmology, Taipei
Veterans General Hospita
Taipei, Taiwan, The Reputh
of China
${ }^{2}$ National Yang-Ming \\ University, Taipei, Taiwan,
The Republic of China \\ ${ }^{3}$ Medical Engineering
Section, National Health \\ Research Institute, Taipei, \\ Taiwan, The Republic of \\ Correspondence: K-H Chen, \\ Department of \\ Hospital, \\ Section II, \\ Tel: 886228757325 ; \\ Fax: 886228761351 \\ E-mail: khchen@
} preventing silicone oil-associated keratopathy. Silicone oil should be removed as early as possible once the goal of tamponade has been (a) 
effects of silicone oil on CEs rather than just acting as a barrier of cell nutrition.

Since we have cultivated human CEs from corneal donors in our laboratory, ${ }_{1}^{17}$ in this study, we tested the toxic effects of silicone oil on the cultured human CEs by evaluating the $\mathrm{CE}$ viability using 3-4,5-dimethylthiazol-2yl)-5-(3-carboxymethoxyphenyl)-2-(4-sulphophenyl)-2Htetrazolium, inner salt (MTS) cell proliferation assay, calcein-acetoxymethyl ester (CAM)-ethidium homodimer staining, and apoptosis assay after exposure to silicone oil in a novel insert well culture system.

\section{Materials and methods}

Optimem-1, Hank's balanced salt solution (Hank's BSS), gentamicin, and trypsin/EDTA were purchased from GibcoBRL/Life Technologies (Rockville, MD, USA). Bovine pituitary fibroblast growth factor (FGF) and nerve growth factor (NGF) were obtained from Biomedical Technologies (Stoughton, MA, USA). Foetal bovine serum (FBS) was obtained from Hyclone (Logan, UT, USA). Epidermal growth factor (EGF) was purchased from Upstate Biotechnologies (Lake Placid, NY, USA). Ascorbic acid, chondroitin sulphate, calcium chloride, ouabain, human lipid fraction, antibiotic/antimycotic solution, and RPMI-1640 multiple vitamin solution were obtained from Sigma (St Louis, MO, USA). Silicone oil of two different viscosities (1000 and 5000 centistoke (CS)), ADATO SIL-OL 1000 and 5000, was purchased from Bausch \& Lomb surgical (Heidelberg, Germany).

\section{Human CE culture}

Fifteen donor corneas of research quality were obtained from National Disease Research Interchange

(Philadelphia, PA, USA), Mississippi Lions Eye Bank, and North Florida Lions Eye Bank. Ages of the donors ranged from 50 to 80 years. This study was performed according to the tenets of the Declaration of Helsinki for research involving human subjects. Each cornea was placed in a Petri dish containing Medium-199 and $50 \mu \mathrm{g} / \mathrm{ml}$ gentamicin. Under a dissecting microscope, Descemet's membrane with the attached endothelium was stripped from the stroma and placed in a $15 \mathrm{ml}$ centrifuge tube containing $0.2 \mathrm{mg} / \mathrm{ml}$ EDTA in Hank's BSS. The tissue was incubated for $1 \mathrm{~h}$ at $37^{\circ} \mathrm{C}$, then triturated with a flame-polished pipette to detach endothelial cells. Cells were pelleted and then resuspended in culture medium containing Optimem- 1 as a basal medium, $8 \%$ foetal bovine serum, $40 \mathrm{ng} / \mathrm{ml} \mathrm{FGF}$, $5 \mathrm{ng} / \mathrm{ml}$ EGF, $20 \mathrm{ng} / \mathrm{ml} \mathrm{NGF}, 20 \mathrm{~g} / \mathrm{ml}$ ascorbic acid, $0.005 \%$ human lipids, $200 \mathrm{mg} / \mathrm{ml}$ calcium chloride, $0.08 \%$ chondroitin sulphate, 1\% RPMI-1640 multiple vitamin solution, $50 \mu \mathrm{g} / \mathrm{ml}$ gentamicin, and antibiotic/ antimycotic solution (diluted $1: 100) .{ }^{17}$ Cells were incubated in six-well tissue culture plates at $37^{\circ} \mathrm{C}$ in a $5 \%$ $\mathrm{CO}_{2}$ humidified atmosphere. Medium was changed on alternate days. Cells reached confluence in 10-14 days. Cells were subcultured after reaching confluence by treating them with trypsin/EDTA and seeding them at a split ratio of $1: 4$ or $1: 8$. Only cells from primary culture or passage 1 were used.

\section{Biocompatibility test of silicone oil}

We tested the biocompatibility of silicone oil and CEs using the method adopted by Malchiodi-Albedi et al. ${ }^{18}$ Briefly, $10^{5}$ cultivated human CEs were seeded onto a poly-L-lysine-coated cell Falcon culture insert with cyclopore bottoms (Becton-Dickinson, Franklin, NJ, USA) and grown in regular culture medium. The inserts were transferred into standard 25-mm wells containing the same medium so that the bottom of the inserts touched the medium. The cultures were maintained in a humidified, $37^{\circ} \mathrm{C}$ incubator with $5 \% \mathrm{CO}_{2}$. On the second day after transfer, the medium on top of the cells was removed and substituted with $1 \mathrm{ml}$ of silicone oil of two different viscosities, 1000 and 5000 CS (Figure 1). In control wells, fresh medium was added. After $48 \mathrm{~h}$, the cultures were evaluated for cell viability including MTS assay, CAM-ethidium homodimer 1 staining, and TUNEL assay. The co-culturing time used in this study, $48 \mathrm{~h}$, is approximately the same as a cell cycle of human CEs. ${ }^{19}$

\section{MTS proliferation bioassay}

After culturing the cells with silicone oil for $48 \mathrm{~h}$, cell viability was assessed by MTS colorimetric assay (Promega Corporation, Madison, WI, USA). MTS is chemically reduced by cells into formazan, which is soluble in tissue culture medium. Absorbance of the formazan was measured at $492 \mathrm{~nm}$. The assay determines dehydrogenase enzyme activity found in metabolically

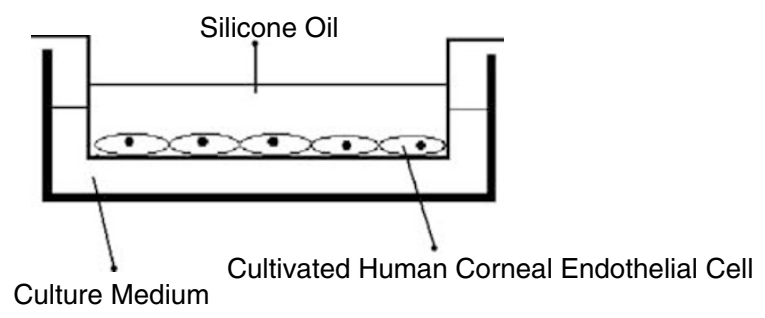

Figure 1 The scheme demonstrates the cultivated corneal endothelial cells (CEs) in the insert well in which the silicone oil is constantly in contact with the CE monolayer on the cell apical side, and in which CEs are grown with the nutrient medium on the basal side. 
active cells. Since the production of formazan is proportional to the number of living cells, the intensity of the produced colour is a good indication of the viability of the cells. Absorbances at $490 \mathrm{~nm}$ (test wavelength) and at $650 \mathrm{~nm}$ (reference wavelength) were measured using an enzyme-linked immunosorbent assay (ELISA) microplate reader (VERSAmax; Molecular Devices, Sunnyvale, CA, USA). Wells containing culture medium but no cells served as controls.

The MTS assay was performed by adding $1 \mathrm{ml}$ of the culture medium and $200 \mu \mathrm{l}$ of MTS to each well. After a 3-h incubation at $37^{\circ} \mathrm{C}$, absorbance at $490 \mathrm{~nm}$ was recorded. Five wells were evaluated for each silicone oil test and each experiment was repeated four times. Statistical significance was analysed by the two-tailed, nonparametric paired $t$-test. $P<0.05$ was considered statistically significant.

\section{Live/dead (CAM-ethidium homodimer 1) staining}

To investigate silicone oil cytotoxicity, human CEs were stained with $2 \mu \mathrm{M}$ CAM and $4 \mu \mathrm{M}$ ethidium homodimer 1 . Live cells were identified by the presence of ubiquitous intracellular esterase activity, which converts the virtually nonfluorescent, cell-permeant CAM to greenfluorescent calcein. Here, ethidium homodimer 1 enters the cells with compromised membranes and, upon binding to nucleic acid, provides a bright red fluorescence in dead cells. Five wells were evaluated for each silicone oil test and each experiment was repeated four times.

After exposure to silicone oil for $48 \mathrm{~h}$ and subsequent removal of the silicone oil, the cultivated human CEs were incubated with a solution containing $2 \mu \mathrm{M}$ CAM and $4 \mu \mathrm{M}$ ethidium homodimer 1 (Molecular Probes, Eugene, OR, USA) for $45 \mathrm{~min}$. Then the cyclopore bottoms of the inserts were carefully cut out and the viability of cultivated human CEs evaluated under a fluorescent microscope (models BH2-RFL-T3 and BX50; Olympus, Tokyo, Japan). To estimate the percentage of dead cell, 10 regions on each well were randomly selected for a total of at least 2000 cells. Dead cell numbers and total cell numbers within each region were counted manually by using a $\times 63$ objective oilimmersion lens. The percentages of dead cells were calculated as the number of dead cells divided by the total number of cells.

\section{Apoptosis assay}

After being exposed to silicone oil, CEs were fixed in $4 \%$ paraformaldehyde in phosphate-buffered saline (PBS) containing $0.12 \mathrm{M}$ of sucrose for $30 \mathrm{~min}$. Apoptotic cells were identified by using TdT-mediated dUTP nick-end labelling (TUNEL) stain with the Dead-End kit (Promega, Madison, WI, USA). TUNEL-positive cells were counted using a Nikon Optiphot microscope. A minimum of eight microscopic fields were chosen randomly, for a total of at least 2000 cells. The percentage of apoptotic cells was calculated as the number of TUNEL-positive cells divided by the total number of cells. Five wells were evaluated for each silicone oil test and each experiment was repeated four times. The cells incubated with $30 \%$ ethanol for $5 \mathrm{~min}$ were used as the positive control (data not shown).

\section{Results}

\section{MTS colorimetric assay}

Enzymatic integrity in cultured human CEs was evaluated with an MTS colorimetric assay. In metabolically active cells, the MTS tetrazolium compound is reduced to a coloured formazan product by a nicotinamide adenine dinucleotide phosphate-or nicotinamide adenine dinucleotide-dependent dehydrogenase. The formazan produced was quantitated with an ELISA microplate reader at $490 \mathrm{~nm}$. Exposure of human CEs to fresh medium in control wells for $48 \mathrm{~h}$ did not alter the dehydrogenase activity. However, when cultured human CEs were exposed to silicone oil for $48 \mathrm{~h}$, dehydrogenase activity was reduced significantly. After co-culturing the cells with $1000 \mathrm{CS}$ silicone oil for $48 \mathrm{~h}$, the dehydrogenase activity decreased to $97.3 \pm 4.4 \%$ (compared to the control well; $P<0.05$ ). When exposed to the higher viscosity (5000 CS) silicone oil, dehydrogenase activity of endothelium cells was significantly suppressed $(81.1 \pm 6.0 \%$ of control value; $P<0.01)$ significantly more than when exposed to the $1000 \mathrm{CS}$ silicone oil (Figure 2; $P<0.05$ ).

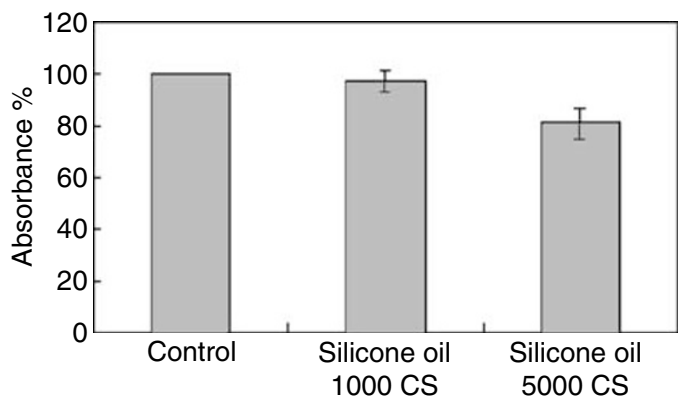

Figure 2 Viability of corneal endothelial cells after silicone oil (1000 and 5000CS) exposure was estimated by the MTS colorimetric assay. In control, cells were incubated in fresh medium only. Values are the mean $\pm S D$ of three separate experiments conducted in quadruplicate. 


\section{Live/dead assay}

After $48 \mathrm{~h}$ of incubation in fresh medium, almost all the human CEs in control wells remained viable, while the cell cytoplasm exhibited intense green fluorescence with the nuclei unstained (Figure 3a). Some of the endothelial cells incubated with 1000-CS silicone oil for $48 \mathrm{~h}$ had red-fluorescent nuclei and only a small amount of cytoplasmic esterase activity (green fluorescence), indicating that the cells were dead. The intense green fluorescence in the cytoplasm of other cells signified their viability (Figure $3 b$ ). The number of cells with red-fluorescent nuclei and little cytoplasmic green fluorescence significantly increased when exposed to the higher viscosity (5000-CS) silicone oil for $48 \mathrm{~h}$ (Figure 3c). The percentage of dead cells was $3.9 \pm 0.1 \%$ (control well), $9.1 \pm 0.1 \%$ (1000-CS

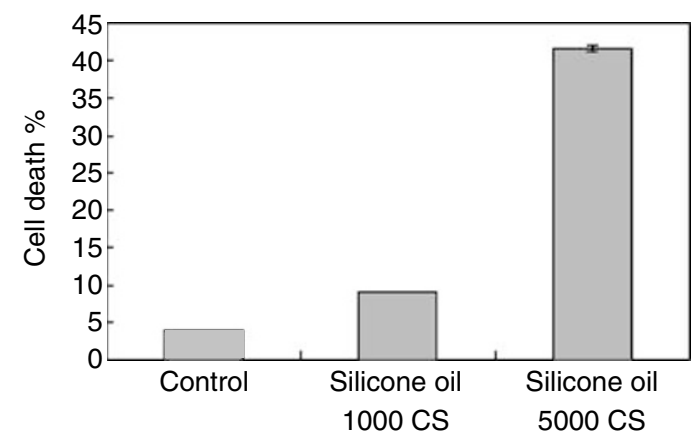

Figure 4 The percentage of dead corneal endothelial cells was determined by counting the cells with red-fluorescent nuclei under CAM-ethidium homodimer 1 staining. Values were the mean $\pm S D$ of three separate experiments conducted in quadruplicate. In each well, at least 2000 cells were evaluated.
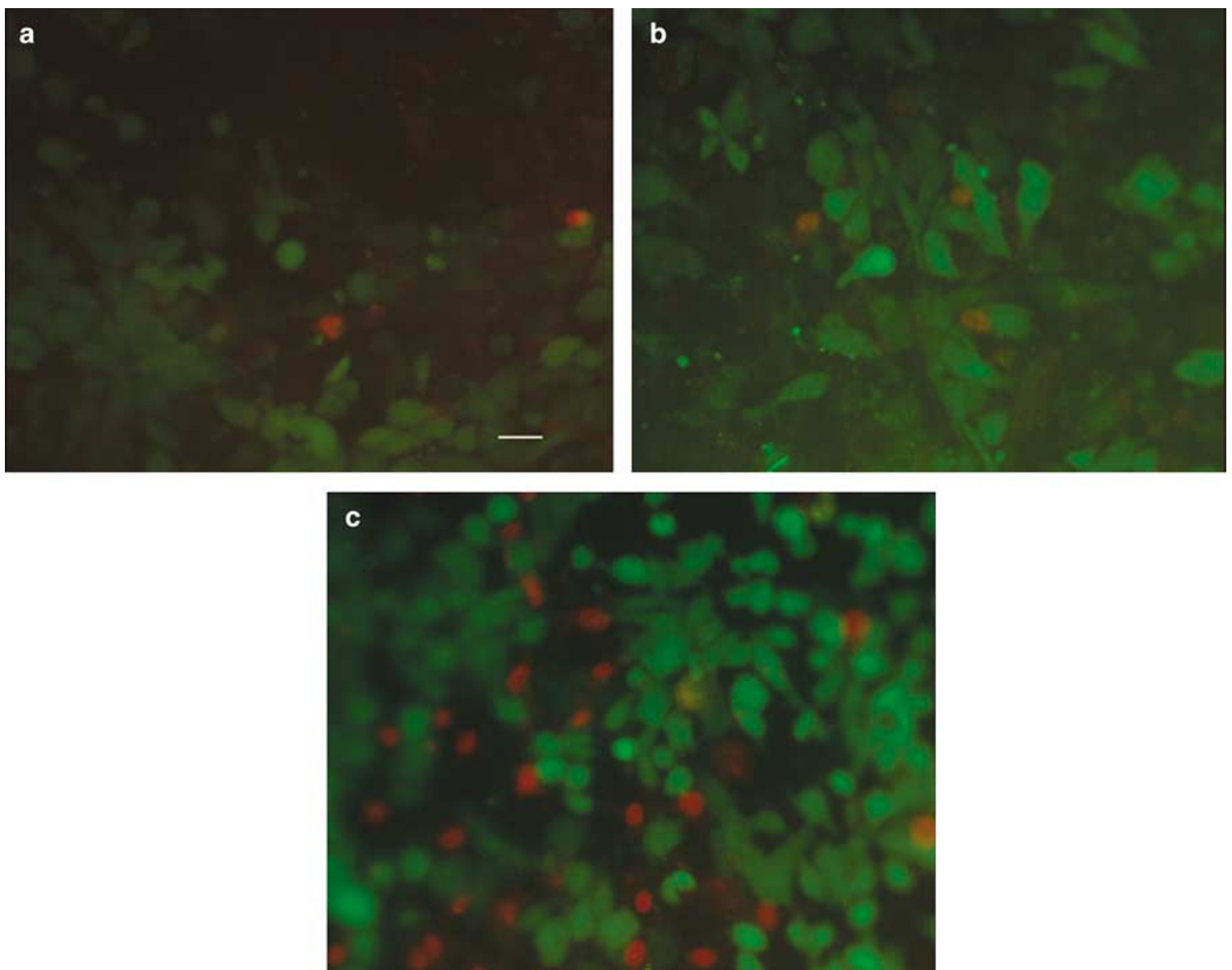

Figure 3 Corneal endothelial cells (CEs) were stained with CAM-ethidium homodimer 1 to assess viability after incubation with silicone oil for $48 \mathrm{~h}$. (a) The CEs were incubated with fresh medium in control well. Almost all the cells showed intense green fluorescence, indicating they were alive. (b) The CEs were incubated in $1000 \mathrm{CS}$ silicone oil for $48 \mathrm{~h}$. Some cells exhibited orange-red fluorescent nuclei with little green fluorescence in the cytoplasm, indicating compromised membrane integrity and reduced esterase activity. Other CEs showed green fluorescence in the cytoplasm and remained viable. (c) The CEs were incubated in 5000 CS silicone oil for $48 \mathrm{~h}$. More cells exhibited orange-red fluorescent nuclei with little green fluorescence in the cytoplasm. Scale bar $=10 \mu \mathrm{m}$. 
silicone oil), and $41.6 \pm 0.4 \%$ (5000-CS silicone oil) (Figure 4). These differences were statistically significant $(P<0.05)$, providing evidence that the silicone oils caused cytotoxicity on human CEs, and that the higher viscosity silicone oil was more toxic.

\section{Apoptosis assay}

Apoptotic cells were identified by using TUNEL labelling with the Dead-End kit. Endothelial cells showing signs of apoptosis, such as condensed and fragmented nuclei or margination of the chromatin to the nuclear membrane, were stained green (Figure 5a). The percentage of apoptotic CEs after being incubated in silicone oil for $48 \mathrm{~h}$ was $1.7 \pm 0.1 \%$ (1000-CS silicone oil) and 9.4 $\pm 0.1 \%$ (5000-CS silicone oil), compared to $0 \%$ (control well) (Figure $5 \mathrm{~b}$ ). These differences were statistically significant $(P<0.05)$.

a
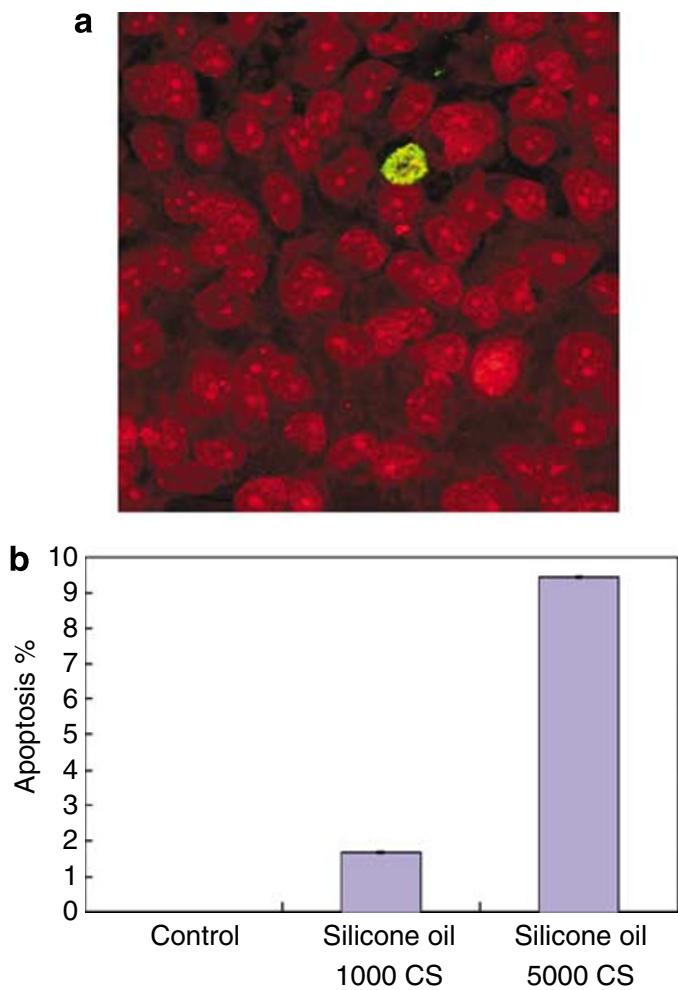

Figure 5 (a) The TUNEL technique showed apoptotic corneal endothelium cell (CEs) after incubation in silicone oil for $48 \mathrm{~h}$ as green fragmented nuclei. Values are the mean \pm SD of three separate experiments conducted in quadruplicate. (b) The percentage of apoptotic CEs (mean \pm SD, percentage) after coculture with silicone oil was determined by counting the cells with green fragmented nuclei by TUNEL staining. Values are the mean $\pm S D$ of three separate experiments conducted in quadruplicate.

\section{Discussion}

In this study, to explore the toxic effect of silicone oil without blocking CEs nutrients, we established an in vitro model in which the silicone oil was constantly in contact with the CE monolayer on the cell apical side in an insert well, and in which CEs were grown with the nutrient medium on the basal side. We demonstrated that contact with silicone oil caused a decrease in cultivated CE proliferation, CE death, and CE apoptosis. Our findings indicated that silicone oil-associated keratopathy may be attributable to the direct cytotoxic effects of silicone oil on human CEs once CE/silicone contact occurred. MTS bioassay showed that silicone oil inhibited CE proliferation. CAM-ethidium homodimer staining revealed some dead endothelial cells after $48 \mathrm{~h}$ silicone oil (1000 and 5000 CS) exposure (9.1 and 41.6\%).

Compared with the dead cells, the percentage of TUNELpositive cells (1.7 and 9.4\%) was relatively low and it might suggest that cell apoptosis plays only a minor role in silicone oil toxicity to CEs.

The literature shows a substantial increase in successful anatomic reattachment of the retina following introduction of intraocular silicone oil tamponade for the treatment of recurrent retinal detachment secondary to proliferative vitreoretinopathy. ${ }^{20,21}$ Unfortunately, visually disabling corneal decompensation is a frequent long-term complication following contact between silicone oil and $\mathrm{CE},{ }^{9,22}$ particularly in aphakic eyes. ${ }^{10}$ However, the mechanism of pathogenesis of CE damage associated with silicone oil is not yet clear. It was believed that corneal contact of silicone oil causes the barrier of nutrition from aqueous humour ${ }^{12}$ and silicone oil was quiet inert in ocular tissues. However, our experiment, exposing cultivated human CEs to silicone oil (apical site of CEs) with nutrition from the culture medium underneath (basal site of CEs) in an insert well system (Figure 1), demonstrated the direct cytotoxicity of silicone oil to CEs without the nutrition supply problems.

Although clinically the silicone oil-associated keratopathy is a long-term side effect, we demonstrate silicone's toxicity to CE in a 48 -h co-culture. Animal studies also showed corneal changes only in days after the injection of silicone oil in anterior chamber. ${ }^{15,16}$ These evidences indicated a possibility of direct toxic effect of silicone oil on CE when they contact. In addition, toxic impurities, the low-molecular-weight ingredients in silicone oil, have been reported to be toxic to CE and may contribute to this toxicity of silicone oil. ${ }^{23,24}$

Our data show the silicone oil with higher viscosity (5000 CS) is more toxic to CE $(P<0.05)$. Silicone oil is water-immiscible and lighter than water (the specific gravity is less than 1.0). The surface tension of silicone oil is higher with its higher viscosity. It has been reported 
that emulsification of silicone oil may produce small oil droplets that could penetrate and injure ocular tissues. ${ }^{25,26}$ However, silicone oil of higher viscosity is believed to emulsify less than the lower viscosity silicone. ${ }^{27,28}$ Therefore, it possibly suggests that the cytotoxicity of silicone oil was not related to emulsification but to the higher surface tension of silicone oil. Further study is needed to clarify the relationship between the viscosity, surface tension, and biocompatibility of silicone oil.

The long-term biocompatibility of intraocular silicone oil is always a concern for the retinal surgeons. In this study, we demonstrated a possibility of the toxicity of silicone oil to human CEs, in addition to a barrier of nutrition from aqueous humour. The prevention of CEssilicone oil contact should help in reducing the chance of silicone oil-associated keratopathy. Therefore, a large, inferiorly located peripheral iridectomy is important to allow aqueous flow into the anterior chamber, and to prevent the forward migration of silicone oil. ${ }^{29}$ Silicone oil should be removed once the goal of tamponade has been achieved and the retinal status is stable.

\section{Acknowledgements}

This study was supported by grants from Taipei Veterans General Hospital (VGH95-C1-144, VGH94-120, VGH94338, VGH94-365-9, and VGHUST94-P1-02), National Health Research Institute (ME-093-CB-\#8, NHRI94A1MEAP01-001), Veterans General Hospitals University System of Taiwan Joint Research Program (VGHUST94P1-02). The medium formulation for growing human corneal endothelial cells has been patented (Growth medium for human corneal endothelial cells; patent no.: US 6,541,256 B1, date of patent: 1 April 2003).

\section{References}

1 McCuen II BW, Azen SP, Stern W, Lai MY, Lean JS, Linton $\mathrm{KL}$ et al. Vitrectomy with silicone oil or perfluoropropane gas in eyes with severe proliferative vitreoretinopathy. Silicone Study Report 3. Retina 1993; 13: 279-284.

$2 \mathrm{Ni} \mathrm{C}$, Wang WH, Albert DM, Schepens CL. Intravitreous silicone injection: histopathologic findings in a human eye after 12 years. Arch Ophthalmol 1983; 101: 1399-1401.

3 Donahue S, Friberg T, Johnson B. Intraconjunctival cavitary inclusions of silicone oil complicating retinal detachment repair. Am J Ophthalmol 1992; 114: 639-640.

4 Eller AW, Friberg TR, Mah R. Migration of silicone oil into the brain: a complication of intraocular silicone oil for retinal tamponade. Am J Ophthalmol 2000; 129: 685-688.

5 Shields C, Eagle R. Pseudo-Schnabel's cavernous degeneration of the optic nerve secondary to intraocular silicone oil. Arch Ophthalmol 1989; 107: 714-717.

6 Shaikh S, Egbert PR, Goldblum RS, Wieland MR. Granulomatous local cell reaction to intravitreal silicone. Arch Ophthalmol 2000; 118: 1133-1134.
7 Budde M, Cursiefen C, Holbach LM, Naumann GO. Silicone oil-associated optic nerve degeneration. Am J Ophthalmol 2001; 131: 392-394.

8 Foulks GN, Hatchell DL, Proia AD, Klintworth GK. Histopathology of silicone oil keratopathy in humans. Cornea 1991; 10: 29-37.

9 Beekhuis WH, van Rij G, Zivojnovic R. Silicone oil keratopathy: indications for keratoplasty. $\mathrm{Br} J$ Ophthalmol 1985; 69: 247-253.

10 Pang MP, Peyman GA, Kao GW. Early anterior segment complications after silicone oil injection. Can J Ophthalmol 1986; 21: 271-275.

11 Hutton WL, Azen SP, Blumenkranz MS, Lai MY, McCuen BW, Han DP et al. The effects of silicone oil removal. Silicone Study Report 6. Arch Ophthalmol 1994; 112: 778-785.

12 Sternberg Jr P, Hatchell DL, Foulks GN, Landers III MB. The effect of silicone oil on the cornea. Arch Ophthalmol 1985; 103: 90-94.

13 Levenson DS, Stocker FW, Georgiade NG. Intracorneal silicone fluid. Arch Ophthalmol 1965; 73: 90-93.

14 Versura P, Cellini M, Torreggiani A, Bernabini B, Rossi A, Moretti $\mathrm{M}$ et al. The biocompatibility of silicone, fluorosilicone and perfluorocarbon liquids as vitreous tamponades. An ultrastructural and immunohistochemical study. Ophthalmologica 2001; 215: 276-283.

15 Norman BC, Oliver J, Cheeks L, Hull DS, Birnbaum D, Green K. Corneal endothelial permeability after anterior chamber silicone oil. Ophthalmology 1990; 97: 1671-1677.

16 Green K, Norman BC, Oliver J, Cheeks L, Hull DS, Birnbaum D. Effects of silicone oils on corneal endothelial permeability. Lens Eye Toxic Res 1990; 7: 413-417.

17 Chen KH, Dimitri A, Joyce NC. Transplantation of adult human corneal endothelial cells - a morphology study. Cornea 2001; 20: 731-737.

18 Malchiodi-Albedi F, Morgillo A, Formisano G, Paradisi S, Perilli R, Scalzo GC et al. Biocompatibility assessment of silicone oil and perfluorocarbon liquids used in retinal reattachment surgery in rat retinal cultures. J Biomed Mater Res 2002; 60: 548-555.

19 Chen KH, Hsu WM, Chiang CC, Li YS. Transforming growth factor-beta2 inhibition of corneal endothelial proliferation mediated by prostaglandin. Curr Eye Res 2003; 26: 363-370.

20 Gonvers M. Temporary silicone oil tamponade in the management of retinal detachment with proliferative vitreoretinopathy. Am J Ophthalmol 1985; 100: 239-245.

21 Zivojnovic R, Mertens DA, Peperkamp E. Liquid silicone in amotio surgery (II). Report on 280 cases - further development of the technic. Klin Monatsbl Augenheilkd 1982; 181: 444-452.

22 Cibis PA, Becker B, Okun E, Canaan S. The use of liquid silicone in retinal detachment surgery. Arch Ophthalmol 1962; 68: 590-599.

23 Green K, Cheeks L, Stewart DA, Trask D. Role of toxic ingredients in silicone oils in the induction of increased corneal endothelial permeability. Lens Eye Toxic Res 1992; 9(3-4): 377-384.

24 Nakamura K, Refojo MF, Crabtree DV, Pastor J, Leong FL. Ocular toxicity of low-molecular-weight components of silicone and fluorosilicone oils. Invest Ophthalmol Vis Sci 1991; 32: 3007-3020.

25 Suzuki M, Okada T, Takeuchi S, Ishii Y, Yamashita H, Hori S. Effect of silicone oil on ocular tissues. Jpn J Ophthalmol 1991; 35: 282-291. 
26 Knorr HL, Seltsam A, Holbach L, Naumann GO. Intraocular silicone oil tamponade. A clinico-pathologic study of 36 enucleated eyes. Ophthalmologe 1996; 93: 130-138.

27 Lakits A, Nennadal T, Scholda C, Knaus S, Gruber H. Chemical stability of silicone oil in the human eye after prolonged clinical use. Ophthalmology 1999; 106: 1091-1100.
28 Heidenkummer HP, Kampik A, Thierfelder S. Experimental evaluation of in vitro stability of purified polydimethylsiloxanes (silicone oil) in viscosity ranges from 1000 to 5000 centistokes. Retina 1992; 12: S28-S32.

29 Ando F. Intraocular hypertension resulting from pupillary block by silicone oil. Am J Ophthalmol 1985; 99: 87-88. 